\section{耍 Heighten Science \\ P U B L I C I T I O N S Corporation ISSN 2639-6653}

\title{
Do you really want to improve the results of treatment for acute pneumonia?
}

\author{
Klepikov Igor* \\ MD, Professor, Renton, Washington, USA
}

\begin{abstract}
*Address for Correspondence: Igor Klepikov MD, Professor, Renton, Washington, USA, Email: igor.klepikov@yahoo.com
\end{abstract}

Submitted: 28 February 2019

Approved: 18 April 2019

Published: 19 April 2019

Copyright: @ 2019 Igor K. This is an open access article distributed under the Creative Commons Attribution License, which permits unrestricted use, distribution, and reproduction in any medium provided the original work is properly cited

Check for updates
The question raised in the title of this letter is a natural consequence of the findings and conclusions that have been growing steadily in recent years regarding the results of treatment for acute pneumonia (AP). If you look at the publications of recent years in this field of medicine, it turns out that one of the main obstacles to progress in improving the results of treatment of this disease is the lack of appropriate methods for determining the pathogen. Thus, the lack of timely diagnostic information about the etiology of the disease excludes the possibility of targeted antibiotic therapy. In recent years, such regrets have become more and more relevant, playing the role of the main explanation for treatment failures .Continuing to narrow the unidirectional view of the problem and to pay attention only to the microbial factor as the main cause of the disease, such views are in fact another illusion, which, even in the case of its hypothetical implementation, will not make significant changes in the overall trend. This statement is easy to verify if you rely on well-known facts, and not use as arguments assumptions and guesses.

First of all, it is necessary to remember the features of the initial period of clinical use of antibiotics. On the one hand, the first results showed their phenomenal effectiveness, and the greatest preference for many years was given to penicillin. On the other hand, bacteriological methods of verification of the pathogen at that time were significantly inferior in their speed and quality indicators to the methods of modern Microbiology. However, treatment of patients with AP for a long time was successfully carried out only by one penicillin and the question of the role of determining the causative agent of inflammation in those years did not have such a critical importance.

I think it should be obvious to readers with medical education that such success in the use of antimicrobials in a dynamic and living biological environment could not continue indefinitely. The further course of events developed according to the so-called law of scissors, but the dynamics of these changes occurred very slowly, which made it difficult to monitor and timely assessment. On the one hand, each new generation of doctors was brought up in the spirit of priority and irreplaceability of antibiotics, gradually forming the image of a kind of panacea. One of the reflections of the new mentality was the wide spread of the principle «antibiotics alone» for the treatment of many diseases (including AP). Declaring this principle of treatment, no one was confused by the fact that the antibiotic in many patients is the only means of treatment and that the same drug is used in radically different processes. And these are indirect signs of the attitude to antibiotics as a panacea. As you know, the basic principle of antibiotics is only intended to suppress the pathogen, is not it? Other medicinal properties of these drugs do not have. All other changes that have already occurred in the focus of inflammation, and in the body in General, can not be eliminated with 
the help of antibiotic therapy, no matter how powerful and long it was not. The latter task falls on individual protection and adaptation mechanisms and depends on the direction of additional assistance methods.

The gradual narrowing of views on the nature of AP to the direct dependence of all sides of the disease on the type of the pathogen was accompanied by the formation of relevant normative documents recommending and prescribing the implementation of specific actions and prescriptions. Today, a specialist working on the basis of a license cannot go beyond these requirements, even if he had doubts about their effectiveness.

In parallel with the deformation of the image of AP as an inflammatory process of nonspecific etiology in the lungs, there is another important process, the results of which are increasingly recorded in recent years. The symbionts of our body are also biological objects. Exposed to mortal danger, they have learned not only to survive, but also to create strains resistant to such aggression.

To date, the group of resistant strains to antibiotics is already quite impressive, and its representatives are increasingly identified among the symbionts of healthy people [1]. In itself, the presence of such microflora in the body does not necessarily mean the fact of the disease, but in the case of the beginning of the last, conventional antibiotic therapy can meet with serious difficulties. In fact, we are talking about microbes that have always been found in the composition of human symbionts and that have only gained additional stability, not aggressiveness. Hardly anyone doubts the fact, that the emergence of such strains is the result of long-term and widespread use of antibiotics. In addition, the frequency of detection of such strains in a healthy population will increase as a result of continued antimicrobial therapy. At the same time, it is even theoretically impossible to stop this already started process, since such an initiative can lead to disastrous results.

Therefore, it is necessary to realistically assess the current situation in bacteriology, which is not the worst option, and consider the gradual increase in the number of carriers of such strains among the healthy population as a future inevitable reality. It is known that the uncertainty of what is happening has always been one of the main prerequisites for the emergence of fears. In this regard, it is especially important to note that the author's point of view is presented to the reader not for emotional impact, but only to develop their own rational assessments of already known facts.

If for many years, the AP microbe is considered to be the main cause of the disease and the entire policy in the treatment of these patients was aimed only at suppressing this factor, the decrease in the effectiveness of treatment and the increase in the resistance of the microflora led scientists into complete confusion. In this case, modern ideas about the nature of the disease can indicate only one way out of this impasse - to speed up the process of determining the pathogen. However, the accumulated evidence shows the futility of trying to realize these hopes.

Although the participation of various microorganisms in the inflammatory process of lung tissue differs significantly in its frequency, and the leaders of this list change periodically, it should be recalled that to date more than 100 microbes have already been established that can act as pathogens of AP [2].

In addition to these data, the literature on this problem always notes the fact that AP pathogens are not only bacteria, but also viruses, fungi, parasites. In this regard, it is especially advisable to pay attention to acute inflammation in the lungs, which is a consequence of a viral infection. According to the statistics of this disease, at least a third of patients in the world have the viral nature of AP $[3,4]$. These indicators cannot be ignored, as the continuation of the therapeutic principle of "antibiotics alone" in the AP reveals the following fact. All patients with viral etiology of the disease do not 
have targeted treatment, and antibiotic therapy is only an attempt to prevent the possible addition of a bacterial component. Therefore, successful treatment in such observations should be considered more as a merit of the patient's body than as a triumph of medical efforts.

At this stage, the etiology of AP is widely interpreted on the basis of the results of bacteriological studies of the microflora of the nasopharynx and oropharynx [5-7]. However, such studies require clarification of the breadth of their application, and the evaluation of their results raises logical objections. For example, it is known that bacteriological examination is usually carried out mainly in hospitalized patients. The etiology of AP in most patients undergoing outpatient treatment remains unknown. But, even among hospitalized patients, the duty of such studies refers to cases of severe forms of the disease, and positive results(including cultures of blood) are found in less than half of the cases $[4,8]$. However, even the interpretation of the positive results of the examination does not explain such a paradox as the presence of less virulent microflora in a number of AP observations compared with bacteriological examination of the upper respiratory tract in healthy people, when the simple presence of aggressive strains without any signs of the disease is revealed.

Serological diagnosis is also not the result of studying the material directly from the area of inflammation. Allowing to identify trace reactions, this type of examination refers to indirect signs of contact of the body with a certain pathogen, but is not an absolute proof of its presence at the moment directly in the inflamed organ. If we continue to discuss the role of the material in the reliability of the results, the most accurate method of determining the agent of AP, of course, is to study the content of the affected area. The possibility of practical implementation of such a study appears only in a limited group of patients with purulent pleural complications that develop at a certain stage of the process and previous treatment. However, even the results of this relatively small group of patients show that in 30 and more $\%$ of cases microflora was not detected [4,9], and there is no reasoned explanation for this fact. As a result, several hundred million AP cases are diagnosed each year in the world, but their etiology remains an open question, and the choice of antimicrobials has been and is being conducted empirically $[4,8,10]$.

Despite the intuitive choice of antimicrobials, their use remains a very important support for the body. For most patients with AP, the suppression of an unexpected mutiny of the accompanying microflora is enough for the body to cope with inflammation in the future. However, in the most severe situations with the rapid and aggressive development of the inflammatory process, another aspect of the problem is revealed, which currently continues to be explained by the extremely high virulence of the pathogen. For example, among patients hospitalized with severe AP, mortality reaches $13-28 \%$.Among the total number of hospitalizations with AP, septic shock is diagnosed in $30 \%$ of cases, and mortality in this group increases to $50 \%$ [4,11-13]. The statistics of these results seem, at first glance, quite understandable and logical: the more severe and aggressive the disease develops, the more difficult it is to eliminate the catastrophic violations that have arisen. However, the logic of such arguments collapses completely, if we take into account some very important, in my opinion, facts.

First, if we consider shock as a septic complication of aggressive forms of AP, it is necessary to have conclusive evidence of this option, the cause of which is the penetration of pathogens into the bloodstream. However, the detection of bacteria in the blood is recorded only in $10-12 \%$ of all hospitalizations with AP without connection with the shock pattern $[8,9]$. That is, the latest figures reflect the frequency of diagnosis of bacteremia, which is not always accompanied by a clinic of shock.

Secondly, interpretations of shock in AP as its septic variant consider this complication only from the point of view of the microflora and leave without attention 
such an important feature of inflammation as an individual reaction of the body to the process. It is the juxtaposition of these two causes (microorganism against macroorganism) that creates the uniqueness of the manifestations of the inflammatory process even in the presence of the same pathogen. However, despite this long-known fact, the severity of AP in recent years is increasingly attributed solely to the properties of microflora.

Finally, the main feature of the nature of the shock in the clinic, the AP determines, from my point of view, the undeniable fact that this intense process is the only inflammatory disease of nonspecific etiology of all known, which occurs in the vascular system of the pulmonary circulation. Rapid change of stages of the process makes it difficult to adapt the body to new conditions. In the most aggressive cases, the body gives us a clear signal of its catastrophic situation. Demonstration of shock in AP is a reflection of the body's efforts to facilitate the work of pulmonary vessels, which could not provide the necessary blood flow and synchronous operation with a large circle of blood circulation [14]. The causes and pathogenesis of this form of shock differ significantly from other variants and require a different treatment solution. So this form of shock has been highlighted by us in a separate section as pulmonal shock. Understanding the pathophysiology of current disorders involves eliminating the reflex effect of the source of inflammation in the lungs on pulmonary blood flow [1518]. Instead, shock in AP is compared with its manifestations of another origin, and such patients begin to receive standard therapy in the form of intravenous injections with the addition of hormones and vasopressors [4,8,9,11,13,19-22].

Intravenous fluids increase blood flow to the pulmonary vessels and further disrupts blood circulation in the small circle, stimulating further hemodynamic changes. In parallel, the processes of exudation in the area of inflammation are stimulated. In response to such aggression, the body of many patients stops responding contrary to expectations and then begins the use of vasopressors and hormones. The result of such therapeutic efforts in pulmonal shock in patients with AP is the loss of many patients, and the frequency of pleural complications among hospitalized patients reaches $60 \%$ or more [4].

The above brief considerations, together with some known facts and figures, allow us to present only the contours of the modern problem of AP treatment and draw the attention of readers to another direction of its solution. It is important to note another fact that a non-standard approach to solving this problem has already been tested in the clinic and its results allowed us to note that early pathogenetically justified assistance with AP guarantees the prevention of a complicated course of the disease $[23,24]$. Therefore, the question that was raised in the title of this letter has a real basis. In order to answer this question in the affirmative, it is necessary first of all to radically reconsider the understanding of the nature of the disease and the mechanisms of its development. Without this step it is impossible to move on.

Do you really want to improve the results of treatment for acute pneumonia? So, go ahead and improve!

\section{References}

1. Davis CP. Normal Flora. In: Baron S, editor. Medical Microbiology. 4th edition. Galveston (TX): University of Texas Medical Branch at Galveston; 1996. Chapter 6. Ref.: https://tinyurl.com/y3tf6qao

2. Ref.: https://tinyurl.com/j2m5ouv

3. Ruuskanen O, Lahti E, Jennings LC, Murdoch DR. Viral pneumonia. Lancet. 2011; 377: 12641275. Ref.: https://tinyurl.com/y47rp7fs

4. AJ Morgan, AJ Glossop. Severe community-acquired pneumonia. BJA Education. 2016: 16: 167-172. Ref.: https://tinyurl.com/y6shz6pd

5. Ref.: https://tinyurl.com/y3dodgln 
6. Jain S, Williams D, Arnold SR, Ampofo K, Bramley AM, et al. Community-Acquired Pneumonia Requieing Hospitalization among US Children. N Engl J Med. 2015; 372: 835-845. Ref.: https://tinyurl.com/y55hup8n

7. Mani CS, Murray DL. Acute Pneumonia and Its Complications. Part II: Clinical Syndromes and Cardinal Features of Infectious Diseases: Approach to Diagnosis and Initial Management. SECTION D: Lower Respiratory Tract Infections. In the book: Long SS, Pickering LK, Prober CG. Principles and Practice of pediatric infectious diseases. Edinburgh, New York: Elsevier Churchill Livingstone. 2012; 235-245. Ref.: https://tinyurl.com/yysca9to

8. Sethi S. Community-Acquired Pneumonia. Merck Manual, professional version. Ref.: https://tinyurl.com/yyq8f7db

9. Waterer GW1, Quasney MW, Cantor RM, Wunderink RG. Septic Shock and Respiratory Failure in Community-acquired Pneumonia Have Different TNF Polymorphism Associations. Am J Respir Crit Care Med. 2001; 163: 1599-604. Ref.: https://tinyurl.com/y5lklvql

10. Prina E, Ranzani OT, Torres A. Community-acquired pneumonia. Lancet. 2015; 386(9998): 1097-108. Ref.: https://tinyurl.com/y247acug

11. Liapikou A, Rosales-Mayor E, Torres A. The management of severe community acquired pneumonia in the ICU. Expert Rev Respir Med. 2014; 8: 293-303. Ref.: https://tinyurl.com/y25e3dgs

12. Vidal A, Santos L. Comorbidities impact on the prognosis of severe acute community-acquired pneumonia. Porto Bio J. 2017: 247-346 Ref.: https://tinyurl.com/y5m2b4jz

13. Ferrer $M 1$,2, Travierso $C 1,3$, Cilloniz $C 1,2$, Gabarrus A1,2, Ranzani OT1, et al. Severe communityacquired pneumonia: Characteristics and prognostic factors in ventilated and non-ventilated patients. PLoS One. 2018; 13: e0191721. Ref.: https://tinyurl.com/y6c9uk9r

14. Klepikov I. Acute Pneumonia is More Cardiovascular than Respiratory Disaster. J Emerg Med Care. 2018; 1: 105. Ref.: https://tinyurl.com/yyhqqhrv

15. Klepikov I. The Meaning of Pulmonary Reflexes in the Pathogenesis of Acute Pneumonia. Intern Med. 2017; 7: 232. Ref.: https://tinyurl.com/y4dflxsx

16. Klepikov I. The Role of Cardiovascular Disorders in the Pathogenesis of Acute Pneumonia. J Cardiol \& Cardiovasc Ther 2017; 4: 1-3. Ref.: https://tinyurl.com/y2e967qh

17. Klepikov I. First Aid for Aggressive Forms of Acute Pneumonia. EC Pulmonology and Respiratory Medicine. 7.2. 2018; 34-37. Ref.: https://tinyurl.com/y2sav66u

18. Klepikov I. Cupping Therapy as a means of First Aid in Acute Pneumonia. J Clin Case Stu. 2018; 3 : Ref.: https://tinyurl.com/y38chvkm

19. Lim TK, Chew MY. Management of severe community acquired pneumonia in the emergency department. J Emerg Crit Care Med. 2018; 2: 2. Ref.: https://tinyurl.com/y43do8tx

20. Angus DC, Barnato AE, Bell D, Bellomo R, Chong CR, et al. A systematic review and meta-analysis of early goal-directed therapy for septic shock: the ARISE, ProCESS and ProMISe Investigators. Intensive Care Med. 2015; 41: 1549-1560. Ref.: https://tinyurl.com/y47zpt7n

21. Kim JW, Kim JJ, Yang HJ, Lim YS, Cho JS, et al. The Prognostic Factors of Pneumonia with Septic Shock in Patients Presenting to the Emergency Department. Korean J Crit Care Med. 2015; 30: 258264 Ref.: https://tinyurl.com/y4a6sut6

22. Klepikov I. The Effect of Intravenous Infusion on the Dynamics of Acute Pneumonia. EC Pulmonology and Respiratory Medicine 4.1. 2017; 15-20. Ref.: https://tinyurl.com/y248b48c

23. Klepikov I. Acute pneumonia and its purulent and destructive complications in children in the midst of a major industrial centre of Western Siberia. Dissertation for the degree of doctor of medical science. Leningrad. 1989.

24. Klepikov I. Acute pneumonia: a new look at the old problem. Lambert Academic Publishing. 2007; 3 : 36. Ref.: https://tinyurl.com/y4m29omu 